\title{
Resolution Characteristics of Holographic Particle Image Velocimetry
}

\author{
J. Scherer* and L. P. Bernal $\dagger$ \\ University of Michigan, Ann Arbor, Michigan 48109
}

\begin{abstract}
The resolution characteristics of in-line holographic imaging systems for application to velocity field measurement in turbulent and other vortical flows has been investigated experimentally. It is shown that the diameter of seed particles strongly influences the maximum size of the test volume that can be used as well as the spatial resolution of the measurement. Velocity measurements in a vortex ring flow using a two-axis holographic recording system are reported. The spatial resolution of this system is approximately $1 \mathrm{~mm}$.
\end{abstract}

\section{Introduction}

$\mathbf{H}$ OLOGRAPHIC imaging of flows seeded with small particles can be a useful technique for velocity field measurement in turbulent and other vortical flows. Several investigators have reported velocity measurements using holographic techniques. ${ }^{1,2}$ Two basic approaches have been proposed. In most cases in-line doubly exposed holograms of the seeded flow are obtained. The velocity is determined from the displacement of the particles on the reconstructed images. This approach is what we call holographic particle image velocimetry (HPIV) because it is a natural extension of planar particle image velocimetry. ${ }^{3}$ A second approach ${ }^{4,5}$ is to obtain a temporal sequence of holograms. The velocity is determined by tracking individual particles as a function of time. The main advantage of HPIV compared to particle tracking techniques is that the particle displacement is determined by local analysis of the reconstructed images which results in reduced image processing requirements.

Turbulent flow measurement requires a large spatial dynamic range. This requirement becomes more severe as the Reynolds number is increased. A large spatial dynamic range requires imaging of very small particles in a large test volume. Research on in-line holographic imaging systems for particle size measurement has shown a limit to the test volume depth of 50-80 far-field distances. ${ }^{6,7}$ (The far-field distance is defined here as $d^{2} / \lambda$ where $d$ is the diameter of the particle and $\lambda$ the wavelength of the laser used to record the hologram.) The depth of field of the reconstructed particle images is of the order of one far-field distance. ${ }^{8}$ The depth of field of the particle image is a good measure of the spatial resolution of the velocity measurement. It follows that the resolution characteristics of the holographic imaging system can severely limit the spatial dynamic range of HPIV.

In this paper we examine experimentally the resolution characteristics of in-line holographic imaging systems. A two-axis holographic recording system designed for high resolution velocity field measurements is described. Finally, velocity measurements in a vortex ring flow using this HPIV system are reported.

Presented as Paper 92-0009 at the AIAA 30th Aerospace Sciences Meeting, Reno, NV, Jan. 6-9, 1992; received March 17, 1992; revision received June 8, 1992; accepted for publication June 12, 1992. Copyright (C) 1992 by the American Institute of Aeronautics and Astronautics, Inc. All rights reserved.

*Graduate Research Assistant, Department of Aerospace Engineering.

†Associate Professor, Department of Aerospace Engineering. Member AIAA.

\section{Holographic Imaging}

The dynamic range requirements of typical HPIV recording systems dictate that the hologram be positioned at a large distance from the particles. In this case the light intensity distribution on the hologram plane is given by Fraunhofer theory ${ }^{9}$

$$
\begin{aligned}
I= & 1-\frac{\pi d^{2}}{2 \lambda z} \sin \left(\frac{\pi r^{2}}{\lambda z}\right)\left[2 J_{1}\left(\frac{\pi d r}{\lambda z}\right) / \frac{\pi d r}{\lambda z}\right] \\
& +\left\{\frac{\pi d^{2}}{4 \lambda z}\left[2 J_{1}\left(\frac{\pi d r}{\lambda z}\right) / \frac{\pi d r}{\lambda z}\right]\right\}^{2}
\end{aligned}
$$

a)
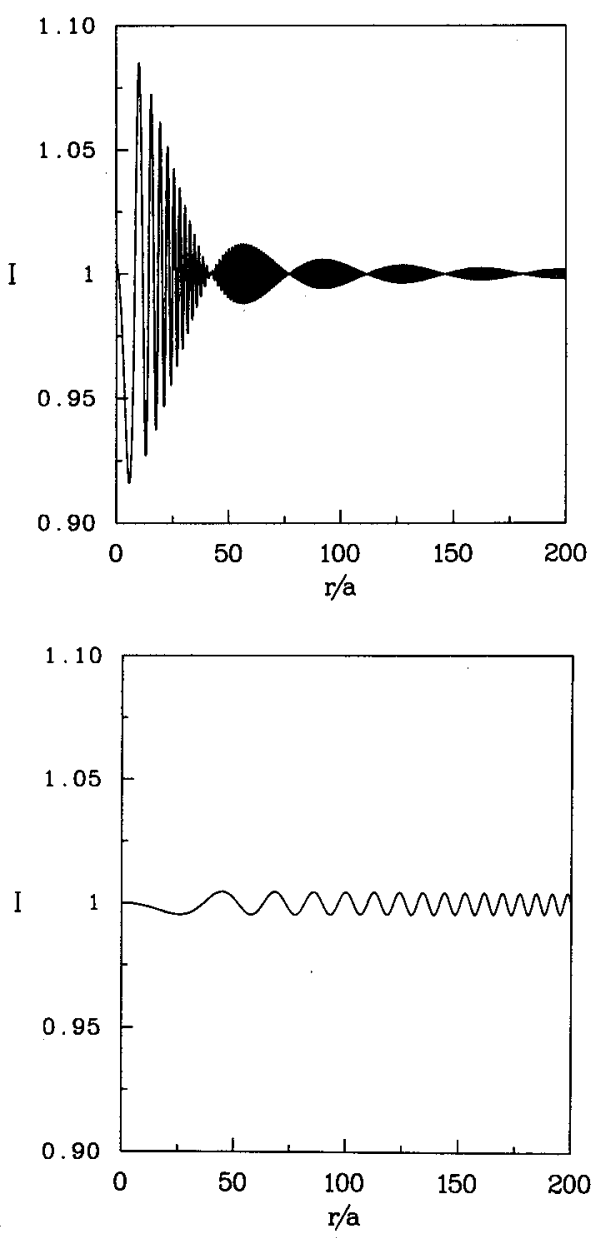

Fig. 1 Calculated intensity distribution on the hologram plane: a) $F=0.058$ and b) $F=0.003$. 


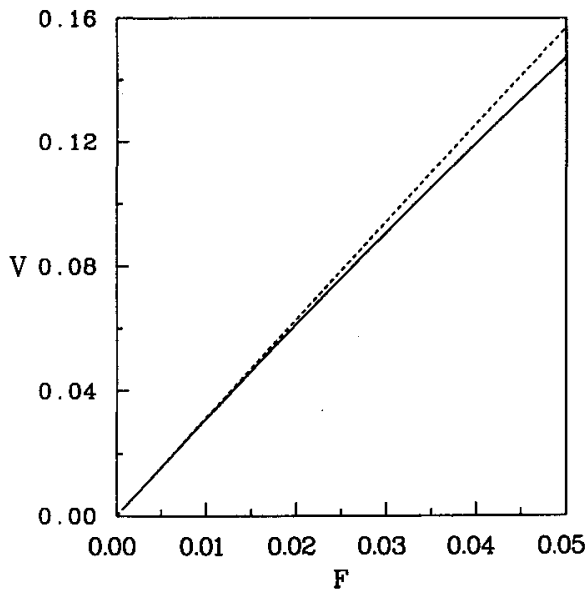

Fig. 2 Fringe visibility as a function of the Fraunhofer number. The solid line corresponds to the exact solution. The broken line is the asymptotic behavior for $F \ll 1$.

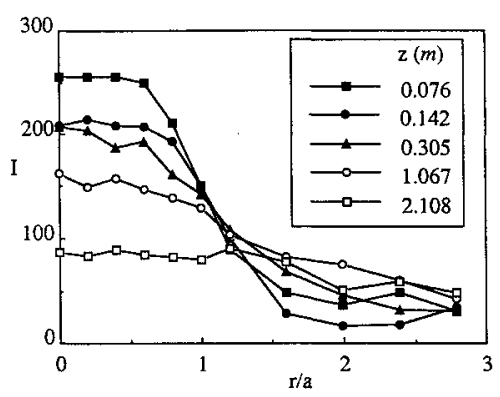

Fig. 3 Intensity distribution on the plane of best focus of the reconstructed image of a 56- $\mu \mathrm{m}$ particle for several hologram recording locations.

where $I$ is the light intensity at a distance $z$ from the center of the particle, and

$$
r=\sqrt{x^{2}+y^{2}}
$$

is the radial distance measured from the optical axis. Typical intensity distributions on the hologram plane calculated from this equation are plotted in Fig. 1. Figure 1a is for a Fraunhofer number $F=d^{2} / \lambda z=0.058$ and Fig. $1 \mathrm{~b}$ for $F=0.003$. These values correspond to typical particle size and hologram positions used in the experiments. In these plots the radial distance $r$ is normalized by the radius of the particle $a$. The high Fraunhofer number case shows a more complicated intensity pattern than the low Fraunhofer number case. In both cases the spatial frequency is not high and can be easily resolved by standard holographic plates. The amplitude of the oscillation is very small. As the Fraunhofer number is decreased the visibility of the fringes is reduced. This effect is illustrated in Fig. 2 where the visibility parameter $V=I_{\max }-I_{\min }$ is plotted as a function of the Fraunhofer number. Here, $I_{\max }$ and $I_{\min }$ are the maximum and minimum values, respectively, of the intensity distribution. For small values of the Fraunhofer number, $V$ approaches the asymptotic behavior $V=\pi F$ shown as a dotted straight line in Fig. 2. The significance of this result is that for a fixed hologram distance, smaller particles produce a weaker fringe pattern or, conversely, for a fixed particle size a greater hologram distance produces a weaker fringe pattern. When the fringe visibility is comparable to the noise level of the reference beam, it will not be possible to differentiate the reconstructed particle image from the background noise.

To document this effect in more detail, a one-axis in-line holographic recording system identical to the one used in the velocity measurements was set up to obtain holograms at sev- eral distances from particles of two diameters. The reconstructed particle images from these holograms were analyzed by measuring the particle intensity distribution at the plane of best focus. The average image intensity was held constant so that meaningful comparison between different cases could be conducted. The results are shown in Figs. 3 and 4 for particles of 56- and $15-\mu \mathrm{m}$ diameters, respectively. As expected, at the same hologram distance the $56-\mu \mathrm{m}$ particles are more sharply defined than the $15-\mu \mathrm{m}$ particles. As the hologram distance is increased, the contrast decreases until the particles can no longer be identified. As discussed earlier, the sharpness of the reconstructed particle images is expected to correlate with the Fraunhofer number. To verify this hypothesis the same data are plotted in Fig. 5 for Fraunhofer numbers $F=0.0058$ and 0.0030 . The results in Fig. 5a at $F=0.0058$ show good collapse of the data which validates the hypothesis. The results at $F=0.0030$ in Fig. 5b show some discrepancy between the two intensity profiles. It should be noted that at this Fraunhofer number the holograms of the $56-\mu \mathrm{m}$ particles were obtained at a distance of over $2 \mathrm{~m}$. This very large distance exceeded the capability of our hologram analysis system. A different reconstruction system was required in this case, which may have contributed to the differences in intensity distribution. At $F=0.0030$ the images of the $15-\mu \mathrm{m}$ particles show good contrast. Based on these measurements we conclude that adequate particle images for HPIV are obtained for $F \geq 0.003$ for moderate hologram distances (up to $1 \mathrm{~m}$ ) which corresponds to a hologram distance of $\mathbf{3 3 3}$ far-field lengths. This is much larger

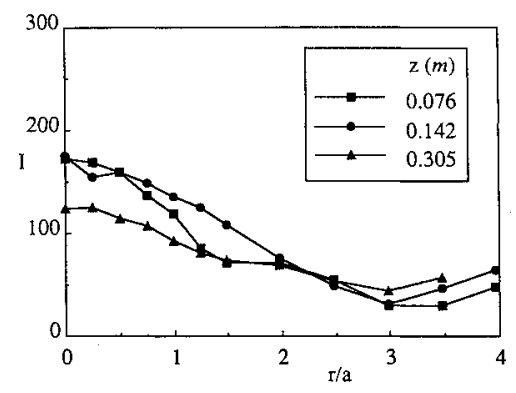

Fig. 4 Intensity distribution on the plane of best focus of the reconstructed image of a $15-\mu \mathrm{m}$ particle for several hologram recording locations.

a)

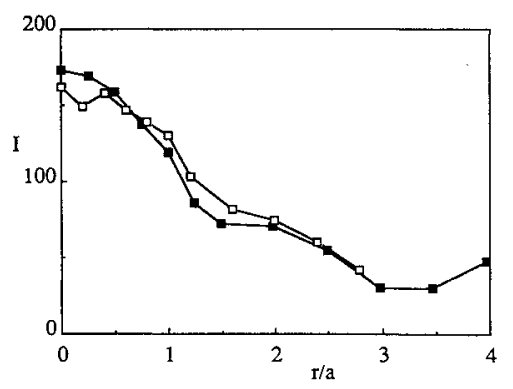

b)

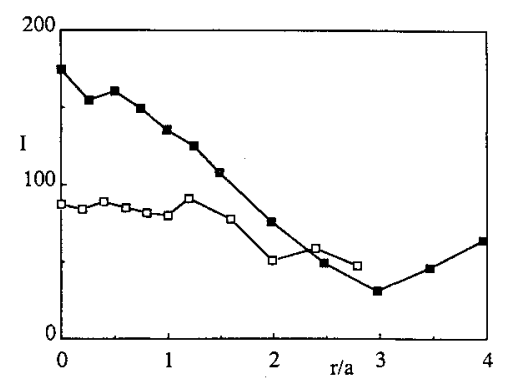

Fig. 5 Intensity distribution on the plane of best focus as a function of Fraunhofer number: a) $F=0.0058$ and b) $F=0.003$; $15-\mu \mathrm{m}$ particle, 56- $\mu \mathrm{m}$ particle. 
than the values reported for particle size measurements. ${ }^{6,7}$ These results are not inconsistent since the images of the particles used for HPIV need not be as sharply defined as for size measurement.

Another important feature of in-line holographic imaging is the depth of field of the reconstructed particle images. The depth of field was determined by measurements of the screen intensity distribution on the reconstructed image of a $44-\mu \mathrm{m}$ particle. The intensity distributions were obtained for various distances $z$, from the location of best focus. The results are presented in Fig. 6. Figure $6 \mathrm{a}$ is the screen intensity distribution as a function of normalized radial distance. Figure $6 \mathrm{~b}$ is the screen intensity distribution as a function of axial distance to best focus. Note the different length scales of the two plots. The characteristic length scale in the radial direction is the particle radius, and the characteristic length scale in the axial direction is the far-field distance $d^{2} / \lambda$. A measure of the depth of field of the particle image is the distance along the particle axis (i.e., $r=0$ ) between points where the intensity level above background has dropped by $50 \%$ compared to the maximum value. These locations are indicated by the broken lines in Fig. $6 \mathrm{~b}$ which are spaced a distance $A=4$ in the horizontal direction. Based on these results the depth of field of the particles is found to be $\pm 2 d^{2} / \lambda$. This is twice the value proposed by Forbes and Kuehn. ${ }^{8}$

\section{Two-Axis Holographic Particle Image Velocity Recording System}

The depth of field of the particle image limits the spatial resolution of the velocity measurement because it determines the distance between particles before they overlap. The depth of field also limits the accuracy of the displacement measurement in the direction of the optical axis. This second effect can be eliminated by using two orthogonal recording systems. To demonstrate this approach, a two-axis in-line holographic recording system was designed and tested in a vortex ring flow field. A schematic diagram of the hologram recording system is shown in Fig. 7. This system uses a copper vapor laser for the light source. A dichroic color separator is used to split the yellow and green lines, one for each optical axis. Two similar optical systems are used to produce collimated beams of 150 $\mathrm{mm}$ diameter intersecting on the test area orthogonal to each other. The collimating optics of each beam incorporates a $100-\mu \mathrm{m}$ spatial filter to improve the spatial coherence of the beam. Commercial holographic plates $(100 \times 125 \mathrm{~mm})$ were used to record the holograms. They were positioned normal to

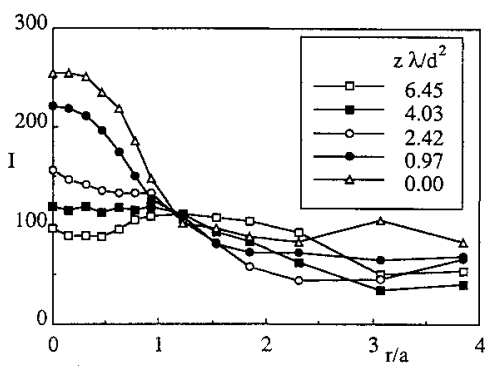

a)

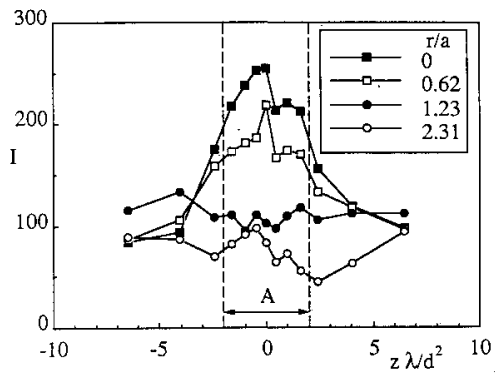

Fig. 6 Intensity distribution for a $44-\mu \mathrm{m}$ particle as a function of a) radial location and b) axial location.

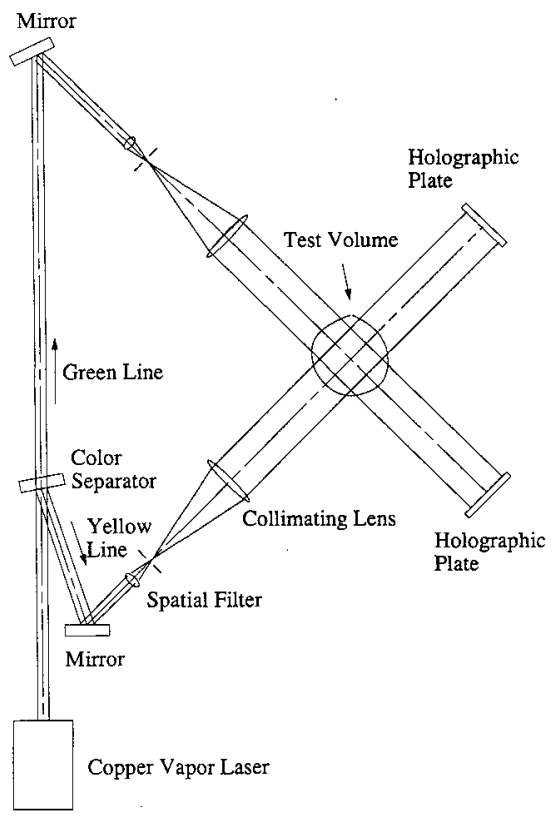

Fig. 7 Schematic diagram of the two-axis in-line holographic recording system.

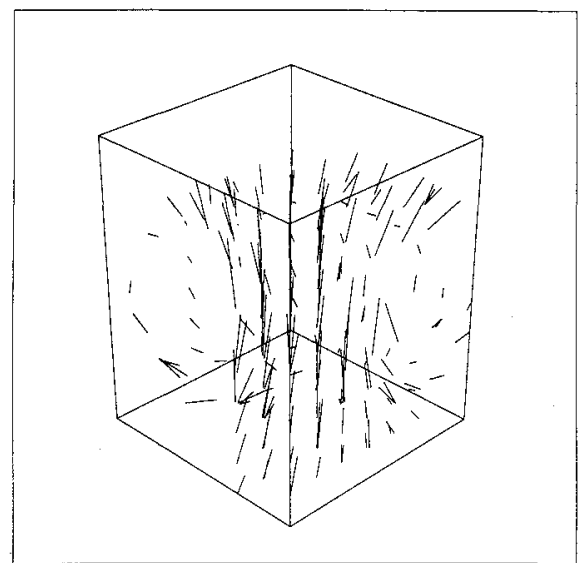

Fig. 8 Velocity vector plot of the measured velocity field in a vortex ring.

the incoming collimated beams at approximately $200 \mathrm{~mm}$ from the center of the test area.

The vortex ring flow was produced in air using a loudspeaker covered by a thin steel plate having a $51-\mathrm{mm}$-circular aperture. The flow was lightly seeded with glass microballoons having a specific gravity of 0.15 and a typical diameter of 15-20 $\mu \mathrm{m}$. To insure that seeding particles were present in the vortical flow, the vortex ring generator was covered and a signal applied to the loudspeaker to mix the seed particles. The flow inside the generator was allowed to decay before firing the vortex ring. As the ring propagated upward through the test area, the copper vapor laser was triggered with a pulse sequence consisting of two pulses followed by three pulses. This allowed the determination of the velocity magnitude and direction from the holograms. The entire firing sequence was automated to obtain consistent operation of the system.

The holograms were analyzed manually using a digital image analysis system. A $5 \mathrm{X}$ microscope objective was used to interrogate a small region of the flow. The velocity of the particles was determined by measuring the displacement on these images with a cursor. One hologram was automatically scanned in $5-\mathrm{mm}$ intervals over a region one-fourth of the entire vortex ring. The displacement information on that hologram (two components) was recorded. This information was then used to scan the second hologram to determine the re- 
maining third component of the displacement. There is redundancy in the system so that one component of the displacement can be measured on both holograms. This information was used to verify that at every point the same particle pair was used to determine the velocity. It should be emphasized that in this system the velocity resolution is determined by the displacement of the particle between light pulses which is of the order of a few hundred microns, much smaller than the depth of field of the particle image. Based on these results it is estimated that a spatial resolution of $1 \mathrm{~mm}$ can be obtained with this system.

A vector plot of the velocity field in the vortex ring is shown in Fig. 8. Because of the low seed concentration used, the velocity was measured in approximately 500 points. No attempt was made to seed the fluid outside the vortex generator. Also, the particles migrate away from the vortex core because of their large density, consequently, velocity measurements were not possible there. The circulation of the vortex ring estimated using these measurements is $\Gamma=420 \mathrm{~cm}^{2} / \mathrm{s}$.

\section{Conclusions}

The resolution characteristics of in-line holographic imaging systems were studied. A two-axis in-line holographic recording system was used to measure the velocity field in a vortex ring flow. The main conclusions of this study are as follows.

The maximum test volume depth was determined experimentally and found to be approximately 300 far-field lengths, $d^{2} / \lambda$.

The spatial resolution of the measurement is determined by the depth of field of the particle images which was found to be $\pm 2 d^{2} / \lambda$.

A two-axis holographic recording system was demonstrated with velocity field measurements in a vortex ring flow. The spatial resolution of this system is of the order of $1 \mathrm{~mm}$.

\section{Acknowledgments}

This research was sponsored by the Office of Naval Research Contract N000184-86-K-0684, URI Program for Ship Hydrodynamics, and N00014-92-J-1058 from the Fluid Dynamics Program.

\section{References}

${ }^{1}$ Trolinger, J., Farmer, W., and Beth, R., "Multiple Exposure Holography of Time Varying Three-Dimensional Fields," Applied Optics, Vol. 7, No. 8, 1968, pp. 1640-1641.

'Schuster, P. R., and Wagner J. W., "Holographic Velocimetry for Flow Diagnostics," Exp. Mech., Vol. 28, No. 4, 1988, pp. 402-408.

${ }^{3}$ Adrian, R. J., "Particle-Imaging Techniques for Experimental Fluid Mechanics," Annual Review of Fluid Mechanics, Vol. 23, 1991, pp. 261-304.

${ }^{4}$ Weinstein, L. M., Beeler, G. B., and Linderman, A. M., "HighSpeed Holocinematographic Velocimeter for Studying Flow Control Physics," AIAA Paper 85-0526, 1985.

${ }_{5}^{5}$ Ruff, G. A., Bernal, L. P., and Faeth, G. M., "High Speed InLine Holocinematography for Dispersed-Phase Dynamics," Applied Optics, Vol. 29, No. 31, 1990, pp. 4544-4546.

${ }^{6}$ Thompson, B. J., Ward, J. H., and Zinky, W. R., "Application of Hologram Techniques for Particle Size Analysis," Applied Optics, Vol. 6, No. 3, 1967, pp. 519-526.

${ }^{7}$ Haussmann, G., and Lauterborn, W., "Determination of Size and Position of Fast Moving Gas Bubbles in Liquids by Digital 3-D Image Processing of Holograms Reconstructions," Applied Optics, Vol. 19, No. 20, 1980, pp. 3529-3535.

${ }^{8}$ Forbes, S. J., and Kuehn, T. H., "In-Line Particle Holography Using Photographic Threshold Technique," Fluids Engineering Division, Vol. 95, American Society of Mechanical Engineers, 1990, pp. 45-47.

${ }^{9}$ Parrent, G. B., Jr., and Thompson, B. J., "On the Fraunhofer (Far Field) Diffraction Patterns of Opaque and Transparent Objects with Coherent Background," Optica Acta, Vol. 11, No. 3, 1964, pp. 183-193. 